\title{
Neural basis of Bilingual Language Control
}

Marco Calabria $^{\mathrm{a}}$, Albert Costa ${ }^{\mathrm{b}, \mathrm{c}}$, David W. Green ${ }^{\mathrm{d}}$, and Jubin Abutalebi ${ }^{\mathrm{e}}$

a. Center for Brain and Cognition, Universitat Pompeu Fabra, Spain

b. Institució Catalana de Recerca i Estudis Avançats (ICREA), Spain

c. Experimental Psychology, Faculty of Brain Sciences, University College London, UK

d. Centre for Neurolinguistics and Psycholinguistics, University Vita-Salute San Raffaele,

Milan, Italy

Corresponding Author:

Albert Costa

Center for Brain and Cognition

Universitat Pompeu Fabra

C. de Ramon Trias Fargas, 25-27 (room 24.329)

08005 Barcelona (Spain)

Phone: (34) 935422602

E-mail: costalbert@gmail.com 


\begin{abstract}
Acquiring and speaking a second language (L2) increases demand on the processes of language control for bilingual as compared to monolingual speakers. Language control for bilingual speakers involves the ability to keep the two languages separated to avoid interference and to select one language or the other in a given conversational context. This ability is what we refer with the term 'bilingual language control' (BLC). It is now well established that the architecture of this complex system of language control encompasses brain networks involving cortical and subcortical structures, each responsible for different cognitive processes such as goal maintenance, conflict monitoring, interference suppression and selective response inhibition. Furthermore, advances have been made in determining the overlap between the BLC network and the non-linguistic executive control (EC) one, under the hypothesis that BLC processes are just an instantiation of a more domain-general control system. Here, we review the current knowledge about the neural basis of these control systems. Results from brain imaging studies of healthy adults and on the performance of bilingual individuals with brain damage are discussed.
\end{abstract}

Keynote: bilingualism, neural basis, language control, bilingual aphasia, executive control 


\section{Language control and bilingualism: an overview}

Why is it so difficult to speak in a second language (L2)? And why is it that bilingual speakers manage to do so without apparent difficulty? A prerequisite for learning an L2 involves acquiring the lexical, phonological and grammatical representations of that language. However, the acquisition of this linguistic knowledge is insufficient for their correct use. Learners of a L2 need to learn how to keep the two languages separated to avoid interference, and learn to select one language or the other in each given communicative situation. We refer to these abilities with the term 'bilingual language control' (BLC).

BLC is often characterized as a set of cognitive processes similarly to those proposed for the domain-general executive control (EC) system. The first model by Abutalebi and Green ${ }^{1}$ described a set of cognitive processes (see earlier Refs. 2 and 3) and related these to specific brain regions interconnected in a network. In bilingual speakers, both languages appear active and compete to control output (for a review, see Ref. 4). Such dual activation necessitates processes that detect and resolve conflict. The processes of detecting and resolving conflict between languages were ascribed to two main brain regions. An anterior cingulate cortex (ACC) region signalled conflict between languages to the prefrontal cortex. This region in turn helped resolve conflict by inhibiting responses from the non-target language and selecting those from the intended one. Other regions contributed with the parietal lobules helping to bias and maintain language selection and subcortical structures of the basal ganglia mediating utterance planning and the selection of the most appropriate lexical alternative. In brief, the BLC network was held to comprise a dorsal anterior cingulate region, the left prefrontal cortex, the left caudate (i.e., basal ganglia) and bilaterally, the inferior parietal lobules. ${ }^{5}$

Green and Abutalebi ${ }^{6}$ offer a more elaborated description of the BLC that is grounded in conversational interactions. They include eight different cognitive processes. They refer to goal maintenance as the process that allows bilingual speakers to establish and maintain the language they want to speak. Goal maintenance requires avoiding potential interference from the non-target languages (with both the self and others as potential sources) and so necessitates the processes of conflict monitoring and interference suppression identified above. A further process is salient cue detection which indicates the need for a language switch in order to align with the new addressee's language. At this point, selective response inhibition stops the speaker from using the current language and initiates the processes of language disengagement and engagement to allow a successful language switch. Finally, the last process described by Green and Abutalebi ${ }^{6}$ is opportunistic planning that they define as the way speakers plan their speech to convey the message.

Green and Abutalebi ${ }^{6}$ elaborated a more extended neural network as a substrate for these processes. This network includes the right prefrontal cortex, the thalamus and the left putamen and the cerebellum. In this review we discuss the contribution of these areas for the control of two languages and how their engagement may be modulated by, for example, the communicative context. The intention to speak one language rather than another requires maintaining use of that language as the goal without being distracted by other cues to the use of the unintended language. In those contexts, in which both languages are used with different interlocutors these two control processes (goal maintenance and interference suppression) are supposed to be more taxed than in those situations in which the speaker needs to stick to just one language. Also, some of these control processes are selectively at play when required by the communicative context: switching back and forth between languages requires processes of engagement and disengagement from one language to another and this is of exclusive relevance in a dual-language context but not in a single-language context. 
A related critical issue to BLC is whether it is just an instantiation of domain-general executive control (EC) processes. This has been a hotly debated issue with models varying in the degree to which they propose the reliance of the BLC system on pre-existing control skills. $^{7-10}$ If BLC is an instantiation of EC process, then bilingualism may affect the development of these set of processes to the extent it imposes additional demand on them compared to that experienced by monolingual speakers. ${ }^{11}$ That is, if there is cross-talk between these two domains then the use of two languages may actually impact domain-general EC processes. This debate has evolved around two issues. The first is whether bilingualism, as compared to monolingualism, confers an advantage or a disadvantage that can be measured behaviourally in tasks involving EC processes. Data speaking to this issue have sparked quite a controversy and, at the moment, the precise conditions under which the bilingual advantage can be found still need to be determined more carefully. We will not touch this issue in our article (for recent reviews, see Refs. 12-15). The second issue refers to the potential changes that bilingualism may exert in certain brain structures related to cognitive control.

In section 2 of this review we describe our current knowledge of how BLC is implemented in the brain and how the implicated neural regions may be modulated by variables related to bilingualism such as the contexts of language use, age of L2 acquisition and L2 proficiency. In section 3, we review the state of the art on the relationship between the neural regions involved in BLC and domain-general EC. Specifically, we review the experimental evidence mostly focusing on brain imaging studies performed with healthy adults and on the performance of individuals with some sort of brain damage (stroke or neurodegenerative diseases). In section 4 we consider the putative neuroprotective effects of bilingualism in healthy aging. We conclude (section 5) with suggestions for further research.

\section{Deconstructing the brain basis of bilingual language control}

The processes of language control act on regions implicated in speech production and comprehension and so shape the outputs of these regions. Figure 1 provides a schematic of the regions involved. In this Figure we simplify the speech pipeline based on Eickhoff et al. ${ }^{16}$ so as to emphasize the control network. Cortically, multiple pathways are known to connect frontal, temporal and parietal regions to support speech production and comprehension. ${ }^{17}$

Indeed, distinct pathways seem to support syntactic, semantic and sensory-motor processes ${ }^{18}$ though how they work together to coordinate sound, syntax and meaning during speech production is under-determined. Our Figure does though acknowledge the importance of subcortical and cerebellar regions to speech production and comprehension (for an overview, see Ref. 19). With this acknowledgement of our simplification, we turn to the neural bases of bilingual language control.

\subsection{The network sustaining BLC: cortical and subcortical structures}

As aforementioned, BLC refers to the set of cognitive abilities that enable bilinguals and multilinguals to use a target language while monitoring for potential interference from language(s) not in use but constantly active., ${ }^{2,3}$ This complex control task is orchestrated by a cortico-subcortical network overlapping with the neural infrastructure for domain-general EC. ${ }^{1,20}$ An intervention of EC in multilingual language processing has been attributed to the need to selectively attend to one language, monitoring speech from other languages' intrusions and switching from one language to another. ${ }^{1,3,21}$

Functional neuroimaging evidence has shown that speakers of more than one language activate regions in neural networks that lie outside the classical perisylvian language network. 
These regions include the dorsolateral prefrontal cortex, the supplementary motor area, the anterior cingulate cortex, the bilateral caudate and the cerebellum. ${ }^{1,22-24}$

Activation in these regions has been consistently detected across varying language pairs such as Chinese-English, ${ }^{25,26}$ Spanish-Catalan ${ }^{27}$ and German-French. ${ }^{28}$ The ACC, and in particular the dorsal ACC/pre-SMA (supplemental motor area), stands out as a critical component mediating the monitoring and suppression of conflicting information, whether in linguistic or in non-linguistic domains. ${ }^{29-33}$

When comparing the conflict resolution abilities of multilingual and monolingual speakers on EC tasks, a lower activation in the ACC of multilinguals was significantly correlated with greater local grey matter volume, suggesting a special tuning of that region for the simultaneous management of multiple languages. ${ }^{34}$ Not surprisingly, the processing demands in multilingual speakers were shown to be particularly taxing in low proficient individuals, who seem to rely heavily on the active suppression of the native language when speaking an L2. In contrast, highly proficient multilinguals would select and maintain their language output with minimal interference from the unintended language(s). ${ }^{1,35}$ Overall, these findings suggest that the control of more than one language is accomplished through a combination of interwoven activities in a neural network that supports not only language processing but also several components of the EC system. We discuss research aimed at dissociating these components in section 3 .

In the following we provide a summary of each neural structure involved in BLC as described in Abutalebi and Green ${ }^{5}$ and the most recent contribution of the adaptive control hypothesis. ${ }^{6,20}$ Figure $1 \mathrm{~B}$ provides an overview of the whole network.

\subsubsection{The anterior cingulate cortex and conflict monitoring}

The ACC has been consistently associated with conflict and error monitoring and its involvement in cognitive control is quite unequivocal. ${ }^{36,37}$ In bilinguals, neural activity of the ACC (and, in particular, the left dorsal ACC/pre-SMA complex) is typically observed during language switching and language selection tasks. ${ }^{1,28,31,32,38-40}$ and for cross-linguistic conflict resolution. ${ }^{41-43}$ However, it is worth underlining that ACC activity is not limited to BLC but is also found in monolinguals for conflict monitoring. Indeed, Abutalebi et al. ${ }^{34}$ reported that bilinguals and monolinguals engage the ACC to the same degree when it comes to monitoring certain language tasks (such as switching between languages for bilinguals and between nouns and verbs for monolinguals). Importantly, for bilinguals the degree to which they engage the ACC is modulated by their language proficiency as shown by Abutalebi et al. ${ }^{34}$ Indeed, for a language with a lower proficiency (which presumably should rely on increased monitoring) bilinguals did engage the ACC more extensively. The important difference between bilinguals and monolinguals is that the former engage the ACC more during everyday life especially when under dual-language circumstances (i.e., in societies where two languages are commonly used). This extra involvement of the ACC (predicted on theoretical grounds) could eventually lead to neurostructural changes such as the observed increased grey matter density in the dorsal ACC, and more efficient use of the ACC on attentional control tasks by bilinguals (for similar findings in aging populations, see Ref. 44).

\subsubsection{The prefrontal cortex and conflict resolution}

The prefrontal cortex has been described as the key player in cognitive control, ${ }^{45}$ especially the dorsolateral prefrontal cortex (DLPFC). ${ }^{46}$ The left prefrontal cortex is commonly activated in linguistic tasks that entail a certain amount of control in bilinguals. ${ }^{28,32,43,47,48}$ Of note, the left prefrontal cortex is highly sensitive to the degree of 
language proficiency and exposure: increased activity is found for an L2 with low proficiency (or less exposure) while less activity is found for a highly mastered L2. ${ }^{4-51}$

As illustrated in our previous model, ${ }^{5}$ the role of the left prefrontal cortex is highly differentiated from that of the ACC with the latter involved in conflict monitoring and the former in conflict resolution. Conflict resolution per se may be subdivided into two distinct cognitive processes: response inhibition and response selection. In our updated model, ${ }^{6,20}$ we further analysed the functional specialization of the prefrontal cortex and illustrated that the left prefrontal cortex is responsible for response selection (such as selecting the correct language in use) and its right counterpart responsible for response inhibition (inhibiting the language not in use). Our assumption was based upon the observation that the right inferior frontal gyrus has been consistently associated to domain-general inhibitory control. ${ }^{52}$ Videsott et al. ${ }^{53}$ provided an interesting observation in this context with multilingual speakers: the right middle frontal gyrus was more engaged during picture naming when naming in the stronger languages (L1 and L2), that afford increase lexical competition, while it was less active during naming in the weaker languages such as L3 and L4 (where supposedly heightened inhibition is less necessary). Related findings were reported by Branzi et al. ${ }^{32}$ who observed that naming in L1 after having named in L2 was significantly associated with increased activity in the right prefrontal cortex while an opposite pattern of activity (i.e., significant deactivation) was found for naming in L2 after having named in L1. One conjecture is that the extra activity observed for naming in L1 after having named in L2 arises because of the necessity to override the inhibition of the prepotent L1 during the previous L2 naming block.

\subsubsection{The inferior parietal lobules and language selection}

In the domain of EC, the inferior parietal lobules are classically linked to attentional tasks ${ }^{54}$ such as bottom-up attentional orienting. ${ }^{55}$ As such, in our model of BLC, we postulated that the inferior parietal lobules would be involved in the attentional aspects of language control: biasing language selection away from the language not in use (left inferior parietal lobule) and biasing selection towards the language in use (right inferior parietal lobule). ${ }^{5}$ Of note, from a historical viewpoint the left inferior parietal lobule has been granted a particular status: it was labelled already in the 1920 s as a so-called multilingual talent area ${ }^{56}$ based upon the observation that lesions to a specific area within the inferior parietal lobule would result into the inability to select languages. Subsequent structural neuroimaging work ${ }^{57,58}$ reported increased grey matter densities for bilinguals as compared to monolinguals in a particular parietal region (the posterior supramarginal gyrus) that links two key components of lexical knowledge: an anterior parietal region that processes phonology, with the angular gyrus that processes meaning. ${ }^{59}$ Further, higher L2 proficiencies in bilinguals correlated with increased grey matter density - a finding most plausibly attributed to an increase in L2 vocabulary knowledge. Other work found increased grey matter density in the same region for multilingual as compared with bilingual speakers, consistent with increased vocabulary knowledge for the former group. ${ }^{60}$

\subsubsection{Subcortical structures}

Subcortical structures are traditionally associated with control functions in the motor, emotional and cognitive domain. ${ }^{61}$ One subcortical nucleus that has been typically linked to BLC is the left caudate nucleus since lesions to this structure result often in a bizarre aphasic pattern characterized by pathological switching between languages, i.e., the inability to speak in one language only (for a review, see Ref. 5). Imaging studies have further confirmed its role in control of two languages ${ }^{1,28,33,62-64}$ in keeping track of the target language ${ }^{39}$ and in the 
control of lexical interference in monolingual speakers during a verbal Stroop task ${ }^{65}$. In contrast to the ACC activity, which seems to be related to domain-general conflict monitoring, left caudate activity may be more related to language proficiency: ${ }^{34}$ the lower the proficiency of a language is, the more left caudate activity is necessary to control that specific language. However, this result is also consistent with a more general role of left caudate in the control of action where it is most active in overcoming habitual action plans ${ }^{66}$ and so is likely triggered in a verbal Stroop task that requires the overcoming of an habitual action - word reading. ${ }^{65}$

The left putamen is another subcortical structure involved in BLC that seems to be related to the control of articulatory processes $^{34}$ (for volumetric increases in this structure, along with the thalamus in simultaneous bilinguals, see Ref. 67) especially in highly demanding language control conditions, such as simultaneous interpreting. ${ }^{68}$

\subsubsection{The cerebellum}

The cerebellum is linked to all the key regions of the language control network. Functional imaging studies using sentence production and comprehension tasks have yet to elucidate the set of cerebellar contributions to language processing. We do know though that cerebellar regions play an important role in both overt speech (e.g., verbal fluency) and inner speech. ${ }^{69}$ For instance, in the case of overt speech grey matter density in bilateral inferior cerebellar regions correlated with the number of words produced during letter and semantic fluency tasks for both L1 and L2. ${ }^{70}$ Moreover, its activation increased when naming in L2 compared to $\mathrm{L1}^{71}$ and during semantic processing in cross-language conditions. ${ }^{72}$ Cerebellar regions may help maintain the representation of a sentence during comprehension and aid in resolving conflicting speech inputs. Indeed, grey matter density in a region of the right cerebellum predicts the facility with which bilingual speakers comprehend an utterance in their second language whilst listening to interfering speech in their first language. ${ }^{73}$

Evidence from brain-damaged patients implicates a fronto-cerebellar circuit in the control of morphosyntax. ${ }^{74}$ Interestingly, in bilingual speakers, grey matter cerebellar volume, though not specifically right cerebellar volume, has been found to correlate with the efficient processing of L2 morphosyntax in immersed, proficient L2 speakers. ${ }^{75}$

\section{FIGURE 1 ABOUT HERE}

\subsection{Modulating the BLC networks}

Various factors may affect the recruitment and adaptive response of the BLC networks. We consider three: age of acquisition of the L2, language proficiency and nature of the language use in bilingual speakers. With respect to the first factor, is there evidence that the simultaneous acquisition of two languages, as opposed to the later, sequential acquisition of a L2 affects the networks of language control? The results of one study by Berken et al. ${ }^{76}$ are consistent with such a possibility though we will suggest that years of use of the two languages is likely to be the critical factor. The Berken et al. research contrasted resting state data in two groups of young adults (aged around 24 years). Each group spoke French and English, with one learning both languages from birth (the simultaneous group) and the other at average at age of 14 years (the sequential group). The groups differed in the strength of resting state functional connectivity amongst the regions involved in language control. It was stronger in the simultaneous group. However, interestingly, within the sequential bilingual group, the earlier the age of L2 learning the stronger connectivity between inferior frontal regions and 
right parietal. Similarly, the modulatory effect of language experience on functional connectivity of frontal-parietal network has been shown in the study by Kousaie et al. ${ }^{77}$ Simultaneous bilinguals had better performance than sequential bilinguals (mean age of acquisition 7.4 years) in a task that measured interference and this behavioural advantage was accompanied by stronger anticorrelation between the attention network and the default mode network.

However, given that the simultaneous group had been using both languages longer than sequential bilinguals, we suggest that resting state functional connectivity may index years of bilingual language use rather the age of L2 acquisition per se. Therefore, the use or the long-term use of the two languages might have changed the functional connectivity of these areas as a result of the interaction between the control regions and language regions (for evidence in bimodal bilinguals, see Ref. 78).

Relative language proficiency is a further factor that modulates some of the regions within the BLC networks when bilinguals switch between languages. The studies that have explored this interaction have found that the variations in relative proficiency (L1 vs. L2 or L3) have found some modulatory effect on the engagement of the pre-supplementary motor area (pre-SMA), ACC, left caudate, and inferior frontal gyrus. Garbin et al. ${ }^{27}$ reported that that switching into the L1 elicited greater activation only in the pre-SMA/ACC complex, while switching into the L2 engaged the left caudate. In contrast, Wang et al. ${ }^{40}$ recorded ACC activation only when bilinguals switched into the low-proficient L2. However, when trilingual speakers have to switch between their most proficient (L1) to their least proficient language (L3), the left caudate shows the greatest increase in activation but the pre-SMA/ACC does not. ${ }^{34}$ Finally, other studies have reported that inferior frontal gyrus together with the preSMA are more activated for language switching to L2 and L3 than to L1 ${ }^{79}$ Though the results are not always consistent across studies they suggest that the relative language proficiency is a key factor that modulates the regional neural response of the BLC network. Possibly, the variations in language proficiency may reflect the differential recruitment of the inhibitory control, as one of the control processes mostly related to the degree of automatisation of language switching. ${ }^{80}$

The processes of BLC are likely to be contingent on the circumstances of use. ${ }^{6,81}$ In some bilingual communities, speakers will switch between their two languages as they address different speakers. Many experimental studies effectively model that kind of circumstance (for a review, see Ref. 82). Despite potential activation, words and structures from the current, non-target language, must be blocked from the intended utterance. In such circumstances, selection of one language involves non-selection of the other: language control is competitive. ${ }^{34}$ The control demands of recurrent patterns of conversational interactions may then shape and tune the networks of language control (Figure 1C). Other communities, by contrast, allow speakers to switch between their languages during an utterance. Indeed, some foster particularly dense codeswitching between languages. We know little about the neural bases of language control in these circumstances. We conjecture that in such circumstances, the relationship between the language networks may be cooperative and allow speakers to recruit words and phrases from either language on an opportunistic basis (Figure 1D). Switching between languages may then not lead to sustained increase in regions such as the left caudate but to an increase demand on the fronto-cerebellar circuit involved in the temporal control of morphosyntax. ${ }^{34,83,84}$

The adaptive language control hypothesis predicts differences in neural response during production when language switching is externally cued, and so requires a competitive control state, compared to during dense codeswitching that induces a cooperative control state. Exploratory research using MEG indeed finds reduced involvement of anterior cingulate and prefrontal cortex involvement during voluntary language switching at least for single word 
production $^{85}$. Additionally, responses of listeners to excerpts of natural codeswitching during conversation revealed only auditory cortex response during a codeswitch. We stress that in some codeswitching communities, the bulk of utterances may be in one language or another ${ }^{86}$ and so language control is likely to be predominantly competitive given the parallel activation of both languages in such contexts.

Overall, considerable progress has been made identifying the networks of language control. The regions involved include cortical, subcortical and cerebellar regions. Future progress is dependent on two factors. First, to detail how the various control processes act within the regions identified. Second, to determine how the BLC network adapts to the control demands of different interactional contexts, such as when bilinguals are in a single- or duallanguage situation or when they codeswitch. Indeed, a recent study by Yang et al. ${ }^{87}$ showed that inhibitory control may be modulated by the language context as revealed by differences in the functional connectivity of the cortical-subcortical-cerebellar circuitry. In Figure 1 we illustrate how the network adapts differently to these different language situations.

\section{Overlap between EC and BLC network}

\subsection{Evidence from healthy individuals}

Besides the indirect evidence of an overlap between BLC and EC, the research now focuses on the direct comparison of brain activation related to linguistic and non-linguistic control tasks in the same populations of bilinguals. ${ }^{88-90}$

To test whether inhibitory control recruits similar areas for the two domains of control, Coderre et al. ${ }^{88}$ compared the brain activation of bilinguals performing a linguistic and a nonlinguistic version of a flanker task. The results of the conjunction analysis revealed a straightforward result: monolinguals did not show any evidence of common brain areas in performing the two tasks, whereas bilinguals showed an overlap in the left inferior frontal gyrus. Similar to the indirect evidence of overlap, the authors concluded that this region is fundamental for both linguistic and non-linguistic control in bilinguals.

However, for switching abilities, such cross-talk appears to be more complex. Weissberger et al ${ }^{89}$ reported similar activation in language switching (naming of numerals) and non-linguistic task switching (naming of colour or shape) in an extensive neural network including cortical and subcortical area. However, when they considered the type of trial (switch or non-switch), they found some differences between linguistic and non-linguistic task activations. For switch trials, language switching, relative to non-linguistic task switching increased activation in the bilateral thalamus, posterior cingulate, right caudate, and cingulate gyrus. For non-switch trials, there was greater activation during the non-linguistic task in frontal, parietal, temporal, occipital, cingulate, insular, and subcortical areas. On the basis of these data, the researchers argued that bilinguals are better at sustaining inhibition of a current non-target language than a current non-target, non-linguistic task. The implication is that though a common network of processes may be involved, the targets of control matter and that the exercise of control may restrict generality.

A similar type of conclusion may be drawn from a study comparing multilingual language switching (using Spanish, Basque, and English) and with multicomponent nonlinguistic switching (using colour, motion, and gender as sorting criteria). ${ }^{90}$ The authors found that the prefrontal cortex (lateral and medial) and the parietal lobule (inferior and superior) were both active in the two tasks. However, as might be expected, the researchers observed differences related to the precise nature of the two tasks. For instance, during language 
switching areas related to phonological processing were more active, whereas dorsal premotor cortex (related to action preparation) was more active during non-linguistic task switching.

In sum, the current evidence of the neural networks of the BLC and the EC systems indicates that the inferior and superior frontal gyri are the best candidates as common brain areas for the general-domain control of inhibition and conflict resolution, whereas the posterior parietal areas for working memory and maintenance of task representations differentiate task-specific processing. ${ }^{1}$ Future research needs to investigate how these brain areas are functionally connected and whether the interaction between the BLC and the EC networks may depend on language context and use. ${ }^{87}$

\subsection{Evidence from brain damage}

There are at least two main reasons why researchers are interested in studying the cross-talk between BLC and EC in pathological populations. First, this helps to know whether control mechanisms in both domains are similarly affected by brain disease, under the hypothesis of their domain-general nature (association of deficits). Second, it has been suggested that selective language impairments, in which one language is more affected that the other, can be explained by deficits of control processes being linguistic or non-linguistic. The hypothesis is based on the idea that the selective loss of one language is due to its overinhibition that results in increasing the threshold for lexical retrieval. $1,5,91-94$

Language control deficits and their relationship with those of the non-linguistic domain have been studied in bilingual patients with aphasia and neurodegenerative diseases.

The more striking problems of language control are described in patients that have pathological language switching and/or pathological language mixing. ${ }^{95}$ In these conditions, patients involuntarily alternate languages across different utterances, or mix elements of two or more languages within a single utterance. ${ }^{96-105}$ These pathological behaviours are due to damage of distinct regions within the BLC network, chief among them subcortical (basal ganglia) and/or frontal areas and their connections with striatal structures. The role of these areas in language switching has been also demonstrated by intraoperative electrical stimulation of the middle frontal gyrus ${ }^{106,107}$ and left caudate. ${ }^{108}$

Although these studies show that specific lesions can dramatically affect language control in bilinguals, it is not clear the extent to which this is paralleled by impairments of EC. This particular relationship has not been yet studied in a systematic way and only a few studies have also investigated the performance of these patients on neuropsychological tests assessing the integrity of the EC system. Some of these studies reported the absence of EC deficits in patients with pathological switching ${ }^{97}$ and some others reported their presence (perseverations, see Ref. 105; "a decrease in general cognitive abilities," see Ref. 99).

Two studies have directly tested the relationship between impairment of linguistic and non-linguistic control functions in this type of patient. ${ }^{101,104}$ Kong et al. ${ }^{104}$ reported the case of a Cantonese-English-Mandarin trilingual speaker (Dr. T) who showed involuntary language switching when speaking in Cantonese. The patient was tested in two EC tasks tapping inhibition and interference (Stroop colour-word test) and shifting abilities (Wisconsin Card Sorting Test). In both tasks, the patient was impaired, leading the authors to conclude that linguistic and non-linguistic control processes are closely related. Similar results were found for the Catalan/Spanish bilingual patient (RM) described by Calabria et al.: ${ }^{101}$ an overlap of deficits common to both domain-general EC and BLC. The authors investigated switching abilities with a language switching task and a non-linguistic switching task. In the language-switching task, RM made several intrusions when required to switch into her dominant language (such as in spontaneous speech), and her accuracy in task switching was significantly impaired compared to age-matched healthy controls. Moreover, her EC deficits 
extended to inhibitory control assessed with a flanker task.

Of note, a second line of research investigating the contribution of EC deficits in language control focuses on bilingual aphasics not necessarily reporting pathological language switching. This type of research is also limited to a few studies, however the interest into it is growing since the landmark observation that concurrent EC deficits may have a negative impact on language recovery in aphasics. ${ }^{109-111}$

Most of the available studies to date have investigated the role of EC in language comprehension by using lexical decision or semantic priming tasks, ${ }^{93,112-114}$ and only a few cases have been studied in the domain of word production. ${ }^{115}$ Moreover, almost all studies have compared linguistic performance to non-linguistic inhibition using a flanker task $^{93,112,113,116}$ or inhibitory control using verbal material. ${ }^{115}$

The two patients described by Green et al. ${ }^{93}$ were compared on linguistic and nonlinguistic control tasks. Patient 1 was impaired on the linguistic control task (Stroop task) but to a lesser degree on the non-linguistic task (flanker) as compared to healthy controls. Patient 2 showed less impairment on linguistic control but more impairment on non-linguistic control. This dissociation of control deficits across domains was interpreted by Green et al. ${ }^{93}$ as differences in the brain regions affected by brain damage. In other words, a lesion in the putamen and globus pallidus (Patient 1) was consistent with damage to the language control device, whereas a lesion within the parietal cortex (Patient 2) was more relevant for the interplay between linguistic and non-linguistic control mechanisms.

Similarly, Dash and Kar ${ }^{112}$ who studied the possible overlap of inhibitory control in linguistic and non-linguistic domains in four aphasic patients found no correlation between performance on the tasks in these two domains. This result led the authors to conclude that the two types of inhibitory control are dissociable, as proposed by Green et al. ${ }^{93}$ In line with this conclusion, Gray and Kiran ${ }^{114}$ found that aphasic patients with semantic deficits did not necessarily show EC deficits (for more evidence of dissociation between control domains, Refs. 113 and 115).

In sum, the evidence gathered from the study of bilingual aphasia suggests that the impact upon a given control process is dependent on its target (linguistic or non-linguistic), in line with the evidence derived from neuroimaging studies. Moreover, those cases yielding a dissociation of deficits across domains are limited to some EC processes such as inhibitory control, leaving unexplored the contribution of others as for instance working memory and updating. One suggestion for future investigations would be to employ neuroimaging techniques to study patients with bilingual aphasia because of the possibility to reveal crossdomain effects of plasticity after language recovery. For instance, some studies have shown increased connectivity within the network involved in language control specifically for the language that was successfully recovered. ${ }^{117,118}$

As to neurodegenerative diseases, some researchers have emphasized that EC may be compromised early on as, for instance, in Parkinson's Disease (PD) (for reviews, see Refs. 119 and 120). Interestingly, these EC deficits have been related to dysfunctions of the basal ganglia and their striatal connections to the frontal cortex. ${ }^{121}$ As aforementioned, these structures and connections are also crucially involved in BLC. ${ }^{5,20,23}$ It follows that studies investigating bilingual individuals with PD may be particularly helpful to test the hypothesis whether BLC and domain general EC are affected to the same degree by brain pathology. ${ }^{122}$

In one of these studies, Zanini et al. ${ }^{123}$ tested Friulian/Italian bilinguals with PD and healthy controls in a sentence comprehension task, syntactic judgment tasks, and an EC task (Wisconsin Card Sorting Test). The authors reported that PD patients were impaired in syntactic processing, particularly in their dominant language (Friulian). Moreover, performance on the EC tasks correlated with performance on the sentence comprehension task suggesting an association between grammatical processing and EC (see also Ref. 124). 
Therefore, this result suggests that EC deficits may contribute to language impairments in bilingual PD patients, possibly because of neurodegeneration of regions (i.e., subcortical regions) that are commonly involved for BLC and EC in bilingual individuals.

Following the idea of exploring the potential overlap of control deficits, Cattaneo et $a l .{ }^{125}$ compared performance on language switching and non-linguistic switching tasks in bilingual individuals with PD and healthy controls. The patients showed greater switch costs than controls for the language switching task but not for non-linguistic switching task. Moreover, the authors also examined the so-called mixing costs (i.e., the difference in reaction times between non-switch trials and single trials, where single trials are ones in a blocked condition involving only a single language, or sorting criterion in the case of the non-linguistic task). Here there was a similar pathology effect for both tasks in individuals with PD. The complexity of these results can be explained in the context of the dual mechanisms of control (DMC) framework proposed by Braver. ${ }^{126}$ This framework postulates two types of control, namely proactive and reactive control with the mixing cost being an index of the first and the switch cost being an index of the second. Accordingly, whereas proactive control may be domain-general, reactive control may be domain-specific. Alternatively, both proactive and reactive control may be domain-general. Under this alternative, switching differences are indicative of differences in the targets of control with increased control demands for the linguistic task.

\section{Bilingualism, healthy aging and neuroprotection}

It has been now widely reported that speaking two or more languages can mitigate the effects of aging upon the human brain. ${ }^{44,127,128}$ Over the lifetime adaptive changes required to represent and control the use of two languages may increase resilience to degenerative changes in the aging brain (for a counter-view based on a meta-analysis of selected retrospective and prospective studies, see Ref. 129). Putative resilience may arise in one of two ways: it may be increased by neural reserve (adaptive changes that lead to more grey and/or white matter volumes or white matter integrity as compared to controls) or by means of neural compensation (to compensate better the loss of neural structure as compared to controls with less loss of brain structure). ${ }^{128}$ Indeed, bilingualism and multilingualism appear to shield against cognitive decline in the elderly ${ }^{130-133}$ and, most strikingly, to delay the onset of dementia, independently of socio-economic status, immigration status or language type, by an average of 4 to 5 years ${ }^{134-137}$ (for a constraint on effectiveness, see Ref. 138). As noted above, evidence in favour of the neuroprotective effects of bilingualism has not gone without challenge. For instance, an analysis of mini-mental state data (MMSE, a measure of global cognitive functioning) in a longitudinal Australian cohort, found that education, but not bilingualism per se was predictive of cognitive decline. ${ }^{129}$ However, neuroprotective effects arguably depend on the precise patterns of bilingual language use ${ }^{6}$ that were not documented in the cohort data. One further source of concern might be that inferences about the long-term neuroprotective effects of bilingualism may reflect the current preponderance of retrospective studies. ${ }^{129}$ However, most of these control for two potential confounders: cultural background and educational level (for a broader discussion of possible counter-evidence and an appraisal of potential confounds, see Refs. 137 and 139).

We have argued that language control in bilingual speakers may exert deep effects on the regions and networks involved in language control. If so, there should be observable structural differences in the brains of older life-time bilingual and monolingual adults. It would then be these differences that provide a reserve against the effects of ageing on the brain. There are few current studies on this issue (for reviews, see Refs. 140 and 141) but their 
results do seem to be in line with what might be expected. Pioneering research on white matter tracts by Luk et al. ${ }^{142}$, for example, revealed that bilingual speakers report greater integrity of long-range tracts in the language network (e.g., the bilateral superior longitudinal fasciculi, right inferior fronto-occipital fasciculus and uncinate fasciculus). Anderson et al. ${ }^{143}$ showed increased white matter integrity specifically in the left superior longitudinal fasciculus for such older life-long bilinguals. Resting state data also distinguished bilingual and monolingual brains with monolinguals showing greater connectivity within frontal regions whereas bilinguals showed enhanced connectivity between the language control regions (left inferior frontal and left caudate) and posterior language regions. These data are interesting because progressive disruption of white matter tracts is typical of the ageing brain. Maintained integrity white-matter tracts, may, as Luk et al. ${ }^{142}$ suggested, compensate for grey matter atrophy (see also Ref. 144) and enhance functional connectivity in bilinguals for the EC network. ${ }^{145}$

Language control processes act on regions of the language network. Grey matter differences might also be predicted between elderly bilingual and monolingual speakers potentially leading to the so-called neural reserve. Indeed, bilateral volumes in the temporal poles and the orbitofrontal cortex were greater for aging bilinguals relative to aged-matched monolinguals, with equivalent scores on education, socio-economic status, and cognitive performance. ${ }^{58,146,147}$ Interestingly, grey matter volumes in the left anterior temporal pole increased with proficiency in the L2 suggesting that language control demands continue to shape the structure of the regions they target. Such a finding is also compatible with a neuroprotective effect of bilingualism, as the temporal poles, along with the orbitofrontal cortex, are among the earliest cortical reasons to that suffer age-related brain atrophy. ${ }^{20}$

Structural neuroimaging studies to date suggest that the life-time exercise of language control in bilingual speakers may lead to adaptive neural changes that are neuroprotective and so provide a basis for the observed 4-5 year delay in the onset of AD symptoms in bilingual speakers. However, in order to explore the causal basis of such neuroprotective effects we need to identify neural differences between the brains of bilingual speakers with AD and those of monolingual speakers with AD. A plausible conjecture is that bilingual individuals will compensate better for any loss of brain structure and function, i.e., display cognitive reserve. In a direct test, Perani et al. ${ }^{148}$ observed relatively better behavioural performance of bilinguals on certain verbal memory tasks combined with greater brain hypometabolism (as measured using positron emission tomography, PET). Most interestingly, the authors also found that the relative use of two languages correlated with increased hypometabolism in posterior brain regions but increased metabolism in regions mediating control (such as the left ACC). Further connectivity analyses strongly suggest that bilinguals with AD compensate by increased metabolic connectivity between posterior to anterior regions: a compensation that reflects the lifelong demand for language control. The idea of an increased connectivity between the posterior to anterior regions fits with the proposal by Grant, Dennis and $\mathrm{Li}^{149}$ that links cognitive control, cognitive and brain reserve in bilingual and aging memory (for a similar hypothesis, see Ref. 150). According to this proposal, and the PASA model, ${ }^{151}$ aging is associated with an increase of frontal activity however in bilinguals there would be a preservation of the posterior parietal activity as usually found in young individuals. Therefore, the integrity of both the posterior and anterior activity coupled with higher connectivity between these two areas would lead to cognitive reserve in bilinguals. 


\section{Conclusion}

The use of two or more languages in daily life poses demands on language control. How neural regions and networks respond to the everyday challenge allows us to explore neuroplastic changes that current research suggests yield neuroprotective benefits in the elderly. Our current understanding derives primarily from controlled experimental studies combined with data from clinical populations. Further advance requires both methodological and technical extensions. Methodologically, we need community-wide agreement on how to characterize differences in bilingual speakers and their profiles of language use and, as is now a common refrain, use suitably powered samples. Technically, there is a need to develop a fuller understanding of neural response during naturalistic exchanges both in terms of comprehension and production because it is these recurrent interactions that shape individual neuroplastic change. Recorded corpora of conversational exchanges from the specific communities of contributing bilingual participants offer a way forward to guide material selection for use in current imaging devices in advance of wearable neuroimaging devices. Finally, as the network sustaining BLC requires the coordination of multiple brain regions, field-based studies (e.g., use of wearable MEG), the use of innovative methods, and analytic techniques will help to better understand its adaptive and dynamic workings. 


\section{Acknowledgment}

The research was supported by grants from the Spanish government (PSI2014-52181-P, and PSI2017-87784-R), the Catalan government (SGR 2009-1521, 2017SGR268), the La Marató de TV3 Foundation (373/C/2014), and the European Research Council under the European Community's Seventh Framework (FP7/ 2007-2013 Cooperation Grant Agreement 613465AThEME). Marco Calabria was supported by the postdoctoral Ramón y Cajal fellowship (RYC2013-14013).

\section{Competing interests}

The authors declare no competing interests.

\section{Figure legend}

\section{Figure 1. The adaptive nature of language control}

The figure illustrates schematized in A) the regions involved in language control (red) and in speech production (blue) and their respective connections, and in B) language control regions overlayed on a brain template. In C) and D) we display two potentially different language contexts in order to highlight the adaptive nature of the language control network. In a situational context where frequently two languages are used, words and structures from the non-target language must be blocked from the intended utterance. In such circumstances, selection of one language involves non-selection of the other and language control is competitive and activates mainly the language control network highlighted in $\mathbf{C}$ ). On the other hand, conversational interactions with particularly dense codeswitching patterns between languages would not rely on the network highlighted in $\mathbf{C}$ ), but on a fronto-cerebellar circuit involved in the temporal control of morphosyntax as highlighted in D). (Modified from Green $\&$ Abutalebi ${ }^{6}$ and Abutalebi \& Green ${ }^{20}$ ). 


\section{References}

1. Abutalebi, J. \& D.W. Green. 2007. Bilingual language production: The neurocognition of language representation and control. J. Neurolinguistics 20: 242-275.

2. Green, D.W. 1986. Control, activation, and resource: a framework and a model for the control of speech in bilinguals. Brain Lang. 27: 210-223.

3. Green, D.W. 1998. Mental control of the bilingual lexico-semantic system. Biling. Cogn. 1: 67-81.

4. Kroll, J.F., P.E. Dussias, K. Bice, et al. 2015. Bilingualism, Mind, and Brain. Annu. Rev. Linguist. 1: 377-394.

5. Abutalebi, J. \& D.W. Green. 2008. Control mechanisms in bilingual language production: Neural evidence from language switching studies. Lang. Cogn. Process. 23: $557-582$.

6. Green, D.W. \& J. Abutalebi. 2013. Language control in bilinguals: The adaptive control hypothesis. J. Cogn. Psychol. 25: 515-530.

7. Costa, A., M. Miozzo \& A. Caramazza. 1999. Lexical selection in bilinguals: Do words in the bilingual's two lexicons compete for selection? J. Mem. Lang. 41: 365-397.

8. Finkbeiner, M., T.H. Gollan \& A. Caramazza. 2006. Lexical access in bilingual speakers: What's the (hard) problem? Biling. Lang. Cogn. 9: 153-166.

9. La Heij, W. 2005. "Selection Processes in Monolingual and Bilingual Lexical Access." In Handbook of bilingualism: Psycholinguistic approaches. J. F. Kroll \& A. M. B. de Groot, Eds.: 289-307. New York, NY, US: Oxford University Press.

10. Mahon, B.Z., A. Costa, R. Peterson, et al. 2007. Lexical selection is not by competition: a reinterpretation of semantic interference and facilitation effects in the picture-word interference paradigm. J. Exp. Psychol. Mem. Cogn. 33: 503-535.

11. Costa, A. \& N. Sebastián-Gallés. 2014. How does the bilingual experience sculpt the brain? Nat. Rev. Neurosci. 15: 336-345.

12. Bialystok, E., J. Abutalebi, T.H. Bak, et al. 2016. Aging in two languages: Implications for public health. Ageing Res. Rev. 27: 56-60.

13. Paap, K.R. \& Z.I. Greenberg. 2013. There is no coherent evidence for a bilingual advantage in executive processing. Cogn. Psychol. 66: 232-258.

14. Valian, V. 2015. Bilingualism and cognition. Biling. Lang. Cogn. 18: 3-24.

15. Yang, H., A. Hartanto \& S. Yang. 2016. The complex nature of bilinguals' language usage modulates task-switching outcomes. Front. Psychol. 7: 560.

16. Eickhoff, S.B., S. Heim, K. Zilles, et al. 2009. A systems perspective on the effective connectivity of overt speech production. Philos. Trans. R. Soc. A Math. Phys. Eng. Sci. 367: 2399-2421.

17. Vigneau, M., V. Beaucousin, P.Y. Hervé, et al. 2006. Meta-analyzing left hemisphere language areas: Phonology, semantics, and sentence processing. Neuroimage 30: 14141432.

18. Friederici, A.D. 2015. White-matter pathways for speech and language processing. In Handbook of Clinical Neurology. Vol. 129 (3rd series) The Human Auditory System G.G. Celesia \& G. Hickok, Eds.: 177-186. Philadelphia, USA: Elsevier.

19. Price, C.J. 2012. A review and synthesis of the first 20years of PET and fMRI studies of heard speech, spoken language and reading. Neuroimage 62: 816-847.

20. Abutalebi, J. \& D.W. Green. 2016. Neuroimaging of language control in bilinguals: neural adaptation and reserve. Biling. Lang. Cogn.19: 689-698.

21. Kroll, J.F., S.C. Bobb, M. Misra, et al. 2008. Language selection in bilingual speech: evidence for inhibitory processes. Acta Psychol. 128: 416-430. 
22. Hervais-Adelman, A.G., B. Moser-Mercer \& N. Golestani. 2011. Executive control of language in the bilingual brain: integrating the evidence from neuroimaging to neuropsychology. Front. Psychol. 2: 234.

23. Luk, G., D.W. Green, J. Abutalebi, et al. 2011. Cognitive control for language switching in bilinguals: A quantitative meta-analysis of functional neuroimaging studies. Lang. Cogn. Process. 27: 1479-1488.

24. Pliatsikas, C. \& G. Luk. 2016. Executive control in bilinguals: A concise review on fMRI studies. Biling. Lang. Cogn. 53: 1689-1699.

25. Guo, T., H. Liu, M. Misra, et al. 2011. Local and global inhibition in bilingual word production: fMRI evidence from Chinese-English bilinguals. Neuroimage 56: 2300-9.

26. Wang, Y., G. Xue, C. Chen, et al. 2007. Neural bases of asymmetric language switching in second-language learners: An ER-fMRI study. Neuroimage 35: 862-870.

27. Garbin, G., A. Costa, A. Sanjuan, et al. 2011. Neural bases of language switching in high and early proficient bilinguals. Brain Lang. 119: 129-135.

28. Abutalebi, J., J.-M. Annoni, I. Zimine, et al. 2008. Language control and lexical competition in bilinguals: an event-related FMRI study. Cereb. cortex 18: 1496-1505.

29. Carter, C.S., M.M. Botvinick \& J.D. Cohen. 1999. The contribution of the anterior cingulate cortex to executive processes in cognition. Rev. Neurosci. 10: 49-57.

30. Hernandez, A.E. 2009. Language switching in the bilingual brain: what's next? Brain Lang. 109: 133-140.

31. Abutalebi, J., P.A. Della Rosa, D.W. Green, et al. 2012. Bilingualism Tunes the Anterior Cingulate Cortex for Conflict Monitoring. Cereb. Cortex 22: 2076-2086.

32. Branzi, F.M., P.A. Della Rosa, M. Canini, et al. 2016. Language control in bilinguals: Monitoring and response selection. Cereb. Cortex 26: 2367-2380.

33. Crinion, J., R. Turner, A. Grogan, et al. 2006. Language control in the bilingual brain. Science 312: 1537-1540.

34. Abutalebi, J., P.A. Della Rosa, G. Ding, et al. 2013. Language proficiency modulates the engagement of cognitive control areas in multilinguals. Cortex 49: 905-911.

35. Costa, A., F.M. Branzi \& C. Avila. 2016. "Bilingualism: Switching." In Neurobiology of Language. Hickok G. \& Small S., Eds.: 419-430. San Diego: Academic Press.

36. Botvinick, M., L.E. Nystrom, K. Fissell, et al. 1999. Conflict monitoring versus selection-for-action in anterior cingulate cortex. Nature 402: 179-181.

37. Botvinick, M.M., T.S. Braver, D.M. Barch, et al. 2001. Conflict monitoring and cognitive control. Psychol. Rev. 108: 624-652.

38. Hosoda, C., T. Hanakawa, T. Nariai, et al. 2012. Neural mechanisms of language switch. J. Neurolinguistics 25: 44-61.

39. Seo, R., A. Stocco \& C.S. Prat. 2018. The bilingual language network: Differential involvement of anterior cingulate, basal ganglia and prefrontal cortex in preparation, monitoring, and execution. Neuroimage 174: 44-56.

40. Wang, Y., P.K. Kuhl, C. Chen, et al. 2009. Sustained and transient language control in the bilingual brain. Neuroimage 47: 414-422.

41. Hsieh, M.C., H. Jeong, K.H. Dos Santos Kawata, et al. 2017. Neural correlates of bilingual language control during interlingual homograph processing in a logogram writing system. Brain Lang. 174: 72-85.

42. Rodriguez-Fornells, A., A. van der Lugt, M. Rotte, et al. 2005. Second language interferes with word production in fluent bilinguals: brain potential and functional imaging evidence. J. Cogn. Neurosci. 17: 422-433.

43. Van Heuven, W.J., K. Conklin, E.L. Coderre, et al. 2011. The Influence of CrossLanguage Similarity on within- and between-Language Stroop Effects in Trilinguals. Front. Psychol. 2: 374. 
44. Borsa, V.M., D. Perani, P.A. Della Rosa, et al. 2018. Bilingualism and healthy aging: Aging effects and neural maintenance. Neuropsychologia 111: 51-61.

45. Miller, E.K. \& J.D. Cohen. 2001. An Integrative Theory of Prefrontal Cortex Function. Annu. Rev. Neurosci. 24: 167-202.

46. Ridderinkhof, K.R., M. Ullsperger, E.A. Crone, et al. 2004. The role of the medial frontal cortex in cognitive control. Science 306: 443-447.

47. Hernandez, A.E., M. Dapretto, J. Mazziotta, et al. 2001. Language switching and language representation in Spanish-English bilinguals: an fMRI study. Neuroimage 14: 510-20.

48. Lehtonen, M.H., M. Laine, J. Niemi, et al. 2005. Brain correlates of sentence translation in Finnish-Norwegian bilinguals. Neuroreport 16: 607-610.

49. Kovelman, I., S.A. Baker \& L.-A. Petitto. 2008. Bilingual and Monolingual Brains Compared: A Functional Magnetic Resonance Imaging Investigation of Syntactic Processing and a Possible "Neural Signature" of Bilingualism. J. Cogn. Neurosci. 20: $153-169$.

50. Jones, O.P., D.W. Green, A. Grogan, et al. 2012. Where, when and why brain activation differs for bilinguals and monolinguals during picture naming and reading aloud. Cereb. Cortex 22: 892-902.

51. Perani, D., J. Abutalebi, E. Paulesu, et al. 2003. The role of age of acquisition and language usage in early, high-proficient bilinguals: An fMRI study during verbal fluency. Hum. Brain Mapp. 19: 170-182.

52. Aron, A.R., T.W. Robbins \& R.A. Poldrack. 2014. Inhibition and the right inferior frontal cortex: One decade on. Trends Cogn. Sci. 18: 177-185.

53. Videsott, G., B. Herrnberger, K. Hoenig, et al. 2010. Speaking in multiple languages: Neural correlates of language proficiency in multilingual word production. Brain Lang. 113: $103-112$.

54. Majerus, S., A. D'Argembeau, T. Martinez Perez, et al. 2010. The Commonality of Neural Networks for Verbal and Visual Short-term Memory. J. Cogn. Neurosci. 22: 2570-2593.

55. Shomstein, S. 2012. Cognitive functions of the posterior parietal cortex: top-down and bottom-up attentional control. Front. Integr. Neurosci. 6: 38.

56. Pötzl, O. 1925. Über die parietal bedingte Aphasie und ihren Einfluss auf das Sprechen mehrer Sprachen. Zeitschrift für die gesamte Neurol. und Psychiatr. 96: 1100-1124.

57. Mechelli, A., J.T. Crinion, U. Noppeney, et al. 2004. Neurolinguistics: Structural plasticity in the bilingual brain. Nature 431: 757.

58. Abutalebi, J., M. Canini, P.A. Della Rosa, et al. 2014. Bilingualism protects anterior temporal lobe integrity in aging. Neurobiol. Aging 35: 2126-2133.

59. Lee, H., J.T. Devlin, C. Shakeshaft, et al. 2007. Anatomical Traces of Vocabulary Acquisition in the Adolescent Brain. J. Neurosci. 27: 1184-1189.

60. Grogan, A., T. 'O. Parker Jones, N. Ali, et al. 2012. Structural correlates for lexical efficiency and number of languages in non-native speakers of English. Neuropsychologia 50: 1347-1352.

61. Graybiel, A.M. 2000. The Basal ganglia. Curr. Biol. 10: R509-11.

62. Price, C.J., D.W. Green \& R. Von Studnitz. 1999. A functional imaging study of translation and language switching. Brain 122: 2221-2235.

63. Zou, L., G. Ding, J. Abutalebi, et al. 2012. Structural plasticity of the left caudate in bimodal bilinguals. Cortex 48: 1197-1206.

64. Branzi, F.M., M. Calabria, M.L. Boscarino, et al. 2016. On the overlap between bilingual language control and domain-general executive control. Acta Psychol. 166: $21-30$. 
65. Ali, N., D.W. Green, F. Kherif, et al. 2010. The Role of the Left Head of Caudate in Suppressing Irrelevant Words. J. Cogn. Neurosci. 22: 2369-2386.

66. Shadmehr, R. \& H.H. Holcomb. 1999. Inhibitory control of competing motor memories. Exp. Brain Res. 126: 235-251.

67. Burgaleta, M., A. Sanjuán, N. Ventura-Campos, et al. 2016. Bilingualism at the core of the brain. Structural differences between bilinguals and monolinguals revealed by subcortical shape analysis. Neuroimage 125: 437-445.

68. Hervais-Adelman, A., B. Moser-Mercer, C.M. Michel, et al. 2015. FMRI of simultaneous interpretation reveals the neural basis of extreme language control. Cereb. Cortex 25: 4727-4739.

69. Tyson, B., C. Lantrip \& R.M. Roth. 2014. Cerebellar contributions to implicit learning and executive functions. Cogn. Sci. 9: 179-217.

70. Grogan, A., D.W. Green, N. Ali, et al. 2009. Structural correlates of semantic and phonemic fluency ability in first and second languages. Cereb. Cortex. 19: 2690-2698.

71. Fu, Y., D. Lu, C. Kang, et al. 2017. Neural correlates for naming disadvantage of the dominant language in bilingual word production. Brain Lang. 175: 123-129.

72. Van de Putte, E., W. De Baene, M. Brass, et al. 2017. Neural overlap of L1 and L2 semantic representations in speech: A decoding approach. Neuroimage 162: 106-116.

73. Filippi, R., F.M. Richardson, F. Dick, et al. 2011. The right posterior paravermis and the control of language interference. J. Neurosci. 31: 10732-10740.

74. Marien, P., S. Engelborghs, F. Fabbro, et al. 2001. The lateralized linguistic cerebellum: A review and a new hypothesis. Brain Lang. 79: 580-600.

75. Pliatsikas, C., T. Johnstone \& T. Marinis. 2014. Grey matter volume in the cerebellum is related to the processing of grammatical rules in a second language: A structural voxel-based morphometry study. Cerebellum 13: 55-63.

76. Berken, J.A., X. Chai, J.-K. Chen, et al. 2016. Effects of Early and Late Bilingualism on Resting-State Functional Connectivity. J. Neurosci. 36: 1165-1172.

77. Kousaie, S., X.J. Chai, K.M. Sander, et al. 2017. Simultaneous learning of two languages from birth positively impacts intrinsic functional connectivity and cognitive control. Brain Cogn. 117: 49-56.

78. Li, L., J. Abutalebi, L. Zou, et al. 2015. Bilingualism alters brain functional connectivity between "control" regions and "language" regions: Evidence from bimodal bilinguals. Neuropsychologia 71: 236-247.

79. De Bruin, A., A. Roelofs, T. Dijkstra, et al. 2014. Domain-general inhibition areas of the brain are involved in language switching: FMRI evidence from trilingual speakers. Neuroimage 90: 348-359.

80. Hut, S.C.A., P. Helenius, A. Leminen, et al. 2017. Language control mechanisms differ for native languages: Neuromagnetic evidence from trilingual language switching. Neuropsychologia 107: 108-120.

81. Green, D. 2011. "Bilingual Worlds." In Language and bilingual cognition. V. Cook, and B. Bassetti, Eds.: 229-240. New York: Psychology Press.

82. Declerck, M. \& A.M. Philipp. 2015. A review of control processes and their locus in language switching. Psychon. Bull. Rev. 22: 1630-1645.

83. Green, D.W. \& L. Wei. 2014. A control process model of code-switching. Lang. Cogn. Neurosci. 29: 499-511.

84. Green, D.W. 2011. Language control in different contexts: The behavioral ecology of bilingual speakers. Front. Psychol. 2: 103.

85. Blanco-Elorrieta, E. \& L. Pylkkänen. 2017. Bilingual language switching in the lab vs. in the wild: The spatio-temporal dynamics of adaptive language control. J. Neurosci. 37: 9022-9036. 
86. Fricke, M. \& G.J. Kootstra. 2016. Primed codeswitching in spontaneous bilingual dialogue. J. Mem. Lang. 91: 181-201.

87. Yang, J., J. Ye, R. Wang, et al. 2018. Bilingual Contexts Modulate the Inhibitory Control Network. Front. Psychol. 9: 395.

88. Coderre, Emily L., F. Smith, Jason, W.J.B. van Heuven, et al. 2015. The functional overlap of executive control and language processing in bilinguals. Biling. (Camb. Engl). 19: 417-488.

89. Weissberger, G.H., T.H. Gollan, M.W. Bondi, et al. 2015. Language and task switching in the bilingual brain: Bilinguals are staying, not switching, experts. Neuropsychologia 66: 193-203.

90. Baene, W. De, W. Duyck, M. Brass, et al. 2015. Brain Circuit for Cognitive Control Is Shared by Task and Language Switching. J. Cogn. Neurosci. 27: 1752-1765.

91. Paradis, M. 2008. Bilingualism and neuropsychiatric disorders. J. Neurolinguistics 21: 199-230.

92. Paradis, M. 1999. Acquired aphasia in bilingual speakers. In Acquired Aphasia (3rd Edition) Taylor Sarno M., Ed. 531-549. London: Academic Press.

93. Green, D.W., A. Grogan, J. Crinion, et al. 2010. Language control and parallel recovery of language in individuals with aphasia. Aphasiology 24: 188-209.

94. Green, D.W. 2008. Bilingual Aphasia: Adapted Language Networks and Their Control. Annu. Rev. Appl. Linguist. 28: 25-48.

95. Ansaldo, A.I. \& K. Marcotte. 2007. Language switching and mixing in the context of bilingual aphasia. In Studying communication disorders in Spanish speakers: Theoretical, research, and clinical aspects. Centeno J.G., Obler L.K., \& Anderson R.T., Eds.:12-21. Cleveland, UK: Multilingual Matters.

96. Aglioti, S. \& F. Fabbro. 1993. Paradoxical selective recovery in a bilingual aphasic following subcortical lesions. Neuroreport 4: 1359-1362.

97. Fabbro, F., M. Skrap \& S. Aglioti. 2000. Pathological switching between languages after frontal lesions in a bilingual patient. J. Neurol. Neurosurg. Psychiatry 68: 650652.

98. Ansaldo, A.I., L.G. Saidi \& A. Ruiz. 2010. Model-driven intervention in bilingual aphasia: Evidence from a case of pathological language mixing. Aphasiology 24: 309324.

99. Mariën, P., J. Abutalebi, S. Engelborghs, et al. 2005. Pathophysiology of language switching and mixing in an early bilingual child with subcortical aphasia. Neurocase 6: $385-398$.

100. Abutalebi, J., M. Miozzo \& S.F. Cappa. 2000. Do subcortical structures control "language selection" in polyglots? Evidence from pathological language mixing. Neurocase 6: 51-56.

101. Calabria, M., P. Marne, L. Romero-Pinel, et al. 2014. Losing control of your languages: a case study. Cogn. Neuropsychol. 31: 266-86.

102. Garcia-Caballero, A., I. Garcia-Lado, J. Gonzalez-Hermida, et al. 2007. Paradoxical recovery in a bilingual patient with aphasia after right capsuloputaminal infarction. $J$. Neurol. Neurosurg. Psychiatry 78: 89-91.

103. Aglioti, S., A. Beltramello, F. Girardi, et al. 1996. Neurolinguistic and follow-up study of an unusual pattern of recovery from bilingual subcortical aphasia. Brain 119: 15511564.

104. Kong A.P.H., J. Abutalebi, K.S.Y. Lam, et al. 2014. Executive and language control in the multilingual brain. Behav. Neurol. 2014: 27951.

105. Leemann, B., M. Laganaro, V. Schwitter, et al. 2007. Paradoxical switching to a barelymastered second language by an aphasic patient. Neurocase case Stud. Neuropsychol. 
neuropsychiatry, Behav. Neurol. 13: 209-213.

106. Sierpowska, J., A. Gabarrós, P. Ripollés, et al. 2013. Intraoperative electrical stimulation of language switching in two bilingual patients. Neuropsychologia 51: 2882-2892.

107. Sierpowska, J., A. Fernandez-Coello, A. Gomez-Andres, et al. 2018. Involvement of the middle frontal gyrus in language switching as revealed by electrical stimulation mapping and functional magnetic resonance imaging in bilingual brain tumor patients. Cortex 99: 78-92.

108. Wang, X., Y.Y. Wang, T. Jiang, et al. 2012. Direct evidence of the left caudate's role in bilingual control: An intra-operative electrical stimulation study. Neurocase 19: 462-469.

109. Kuzmina, E. \& B.S. Weekes. 2017. Role of cognitive control in language deficits in different types of aphasia. Aphasiology 31: 765-792.

110. Murray, L.L. 2012. Attention and other cognitive deficits in aphasia: Presence and relation to language and communication measures. Am. J. Speech-Language Pathol. 21: S51-64.

111. Murray, L. 2017. Focusing attention on executive functioning in Aphasia. Aphasiology 31: 721-724.

112. Dash, T. \& B.R. Kar. 2014. Bilingual language control and general purpose cognitive control among individuals with bilingual aphasia: Evidence based on negative priming and flanker tasks. Behav. Neurol. 2014: 679706.

113. Verreyt, N., M. De Letter, D. Hemelsoet, et al. 2013. Cognate Effects and Executive Control in a Patient With Differential Bilingual Aphasia. Appl. Neuropsychol. 20: 221230.

114. Gray, T. \& S. Kiran. 2015. The relationship between language control and cognitive control in bilingual aphasia. Biling. Lang. Cogn. 19: 1-20.

115. Faroqi-Shah, Y., M. Sampson, M. Pranger, et al. 2016. Cognitive control, word retrieval and bilingual aphasia: Is there a relationship? J. Neurolinguistics 45: 95-109.

116. Gray, T. \& S. Kiran. 2013. A theoretical account of lexical and semantic naming deficits in bilingual aphasia. J. Speech, Lang. Hear. Res. 56: 1314-1327.

117. Radman, N., M. Mouthon, M. Di Pietro, et al. 2016. The Role of the Cognitive Control System in Recovery from Bilingual Aphasia: A Multiple Single-Case fMRI Study. Neural Plast. 2016: 8797086.

118. Abutalebi, J., P.A. Della Rosa, M. Tettamanti, et al. 2009. Bilingual aphasia and language control: A follow-up fMRI and intrinsic connectivity study. Brain Lang. 109: 141-156.

119. Dirnberger, G. \& M. Jahanshahi. 2013. Executive dysfunction in Parkinson's disease: a review. J. Neuropsychol. 7: 193-224.

120. Kudlicka, A., L. Clare \& J. V Hindle. 2011. Executive functions in Parkinson's disease: Systematic review and meta-analysis. Mov. Disord. 26: 2305-2315.

121. Narayanan, N.S., R.L. Rodnitzky \& E.Y. Uc. 2013. Prefrontal dopamine signaling and cognitive symptoms of Parkinson's disease. Rev. Neurosci. 24: 267-78.

122. Calabria, M., G. Cattaneo \& A. Costa. 2017. It is time to project into the future: "Bilingualism in healthy and pathological aging." J. Neurolinguistics 43: 1-3.

123. Zanini, S., A. Tavano, L. Vorano, et al. 2004. Greater syntactic impairments in native language in bilingual Parkinsonian patients. J. Neurol. Neurosurg. Psychiatry 75: 1678-1681.

124. Johari, K., F. Ashrafi, A. Zali, et al. 2013. Grammatical deficits in bilingual Azari Farsi patients with Parkinson's disease. J. Neurolinguistics 26: 22-30.

125. Cattaneo, G., M. Calabria, P. Marne, et al. 2015. The role of executive control in 
bilingual language production: A study with Parkinson's disease individuals. Neuropsychologia 66: 99-110.

126. Braver, T.S. 2012. The variable nature of cognitive control: A dual-mechanisms framework. Trends Cogn. Sci. 16: 106-113.

127. Bialystok, E., F.I.M. Craik, D.W. Green, et al. 2010. Bilingual minds. Psychol. Sci. 10: 89-129.

128. Perani, D. \& J. Abutalebi. 2015. Bilingualism, dementia, cognitive and neural reserve. Curr. Opin. Neurol. 28: 618-625.

129. Mukadam, N., A. Sommerlad \& G. Livingston. 2017. The Relationship of Bilingualism Compared to Monolingualism to the Risk of Cognitive Decline or Dementia: A Systematic Review and Meta-Analysis. J. Alzheimer's Dis. 58:45-54.

130. Bak, T.H., J.J. Nissan, M.M. Allerhand, et al. 2014. Does bilingualism influence cognitive aging? Ann. Neurol. 75:959-63.

131. Wilson, R.S., P.A. Boyle, J. Yang, et al. 2015. Early life instruction in foreign language and music and incidence of mild cognitive impairment. Neuropsychology 29: 292-302.

132. Kavé, G., N. Eyal, A. Shorek, et al. 2008. Multilingualism and Cognitive State in the Oldest Old. Psychol. Aging 23:70-78.

133. Perquin, M., M. Vaillant, A.M. Schuller, et al. 2013. Lifelong Exposure to Multilingualism: New Evidence to Support Cognitive Reserve Hypothesis. PLoS One 8: e62030.

134. Alladi, S., T.H. Bak, V. Duggirala, et al. 2013. Bilingualism delays age at onset of dementia, independent of education and immigration status. Neurology 81:1938-1944.

135. Bialystok, E., F.I. Craik, R. Klein, et al. 2004. Bilingualism, aging, and cognitive control: evidence from the Simon task. Psychol. Aging 19: 290-303.

136. Woumans, E., P. Santens, A. Sieben, et al. 2015. Bilingualism delays clinical manifestation of Alzheimer's disease. Biling. Lang. Cogn. 18: 568-574.

137. Woumans, E., J. Versijpt, A. Sieben, et al. 2017. Bilingualism and Cognitive Decline: A Story of Pride and Prejudice. J. Alzheimer's Dis. 60:1237-1239.

138. Gollan, T.H., D.P. Salmon, R.I. Montoya, et al. 2011. Degree of bilingualism predicts age of diagnosis of Alzheimer's disease in low-education but not in highly educated Hispanics. Neuropsychologia 49: 3826-3830.

139. Bak, T.H. 2016. The impact of bilingualism on cognitive ageing and dementia: Finding a path through a forest of confounding variables. Linguist. Approaches to Biling. 6: 205226.

140. Gold, B.T. 2015. Lifelong bilingualism and neural reserve against Alzheimer's disease: A review of findings and potential mechanisms. Behav. Brain Res. 281: 281-289.

141. Perani, D. \& J. Abutalebi. 2005. The neural basis of first and second language processing. Curr. Opin. Neurobiol. 15: 202-206.

142. Luk, G., E. Bialystok, F.I. Craik, et al. 2011. Lifelong bilingualism maintains white matter integrity in older adults. J. Neurosci. 31: 16808-16813.

143. Anderson, J.A.E., J.G. Grundy, J. De Frutos, et al. 2018. Effects of bilingualism on white matter integrity in older adults. Neuroimage 167: 143-150.

144. Gold, B.T., N.F. Johnson \& D.K. Powell. 2013. Lifelong bilingualism contributes to cognitive reserve against white matter integrity declines in aging. Neuropsychologia 51: 2841-2846.

145. Grady, C.L., G. Luk, F.I.M. Craik, et al. 2015. Brain network activity in monolingual and bilingual older adults. Neuropsychologia 66: 170-181.

146. Olsen, R.K., M.M. Pangelinan, C. Bogulski, et al. 2015. The effect of lifelong bilingualism on regional grey and white matter volume. Brain Res. 1612: 128-139.

147. Li, L., J. Abutalebi, K. Emmorey, et al. 2017. How bilingualism protects the brain from 
aging: Insights from bimodal bilinguals. Hum. Brain Mapp. 38: 4109-4124.

148. Perani, D., M. Farsad, T. Ballarini, et al. 2017. The impact of bilingualism on brain reserve and metabolic connectivity in Alzheimer's dementia. Proc. Natl. Acad. Sci. 114:1690-1695.

149. Grant, A., N.A. Dennis \& P. Li. 2014. Cognitive control, cognitive reserve, and memory in the aging bilingual brain. Front. Psychol. 5: 1401.

150. Grundy, J.G., J.A.E. Anderson \& E. Bialystok. 2017. Neural correlates of cognitive processing in monolinguals and bilinguals. Ann. N. Y. Acad. Sci. 1396: 183-201.

151. Davis, S.W., N.A. Dennis, S.M. Daselaar, et al. 2008. Que PASA? The posterior anterior shift in aging. Cereb. Cortex 18:1201-9

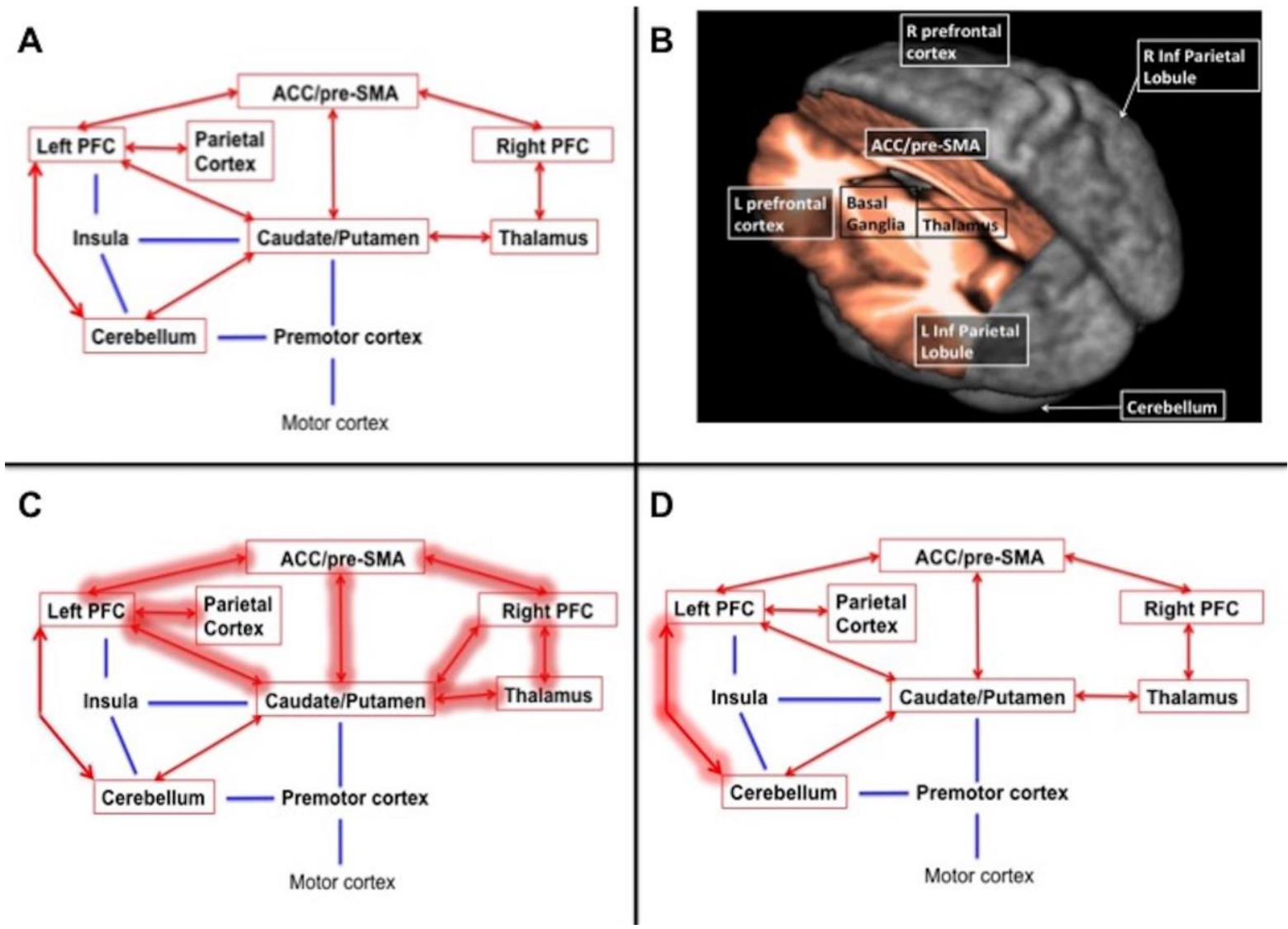

